\title{
Interpolation by bivariate polynomials based on Radon projections
}

\author{
by \\ B. Bojanov and I. K. Georgieva (Sofia)
}

\begin{abstract}
For any given set of angles $\theta_{0}<\ldots<\theta_{n}$ in $[0, \pi)$, we show that a set of $\left(\begin{array}{c}n+2 \\ 2\end{array}\right)$ Radon projections, consisting of $k$ parallel $X$-ray beams in each direction $\theta_{k}$, $k=0, \ldots, n$, determines uniquely algebraic polynomials of degree $n$ in two variables.
\end{abstract}

1. Introduction. Most of the methods for approximate reconstruction of a univariate function $f$ are based on sampling values of $f$ at a finite number of points, and the tools used are usually those of interpolation. This is a natural approach to approximation of univariate functions since a table of function values is a standard type of information about $f$ that comes as output in practical problems and processes described by functions in one variable, and in addition, the Lagrange interpolation problem by polynomials is always solvable. In the multivariate case, such an approach encounters serious difficulties. For example, it is well known that pointwise interpolation by multivariate polynomials is no more possible for every choice of nodes. Moreover, there are a lot of practical problems in which information about the relevant function comes as a set of functionals different from point evaluations. In tomography, electronic microscopy, and technics, the data often consists of values of linear integrals over segments. In many situations, a table of mean values of a function of $d$ variables on $(d-1)$-dimensional hyperplanes is considered to be the most natural type of data for multivariate functions. Hakopian's famous interpolation formula [6] (see also [7]) is an important reason to take this approach.

Hakopian proved that for any given $n+2$ distinct points $X_{0}, \ldots, X_{n+1}$ on the boundary of a convex body $D$ (say, a disk), the set of integrals of $f$ over all the linear segments $\left[X_{i}, X_{j}\right]$ determines uniquely every polyno-

2000 Mathematics Subject Classification: 41A05, 41A63.

The research of the first author was supported by the Banach Center of IM PAN, Warsaw, and partially by the Bulgarian Ministry of Science under Contract No. MM$802 / 98$. 
mial $f$ of degree $n$. This is actually a particular case $(d=2)$ of a more general multivariate result he proved in [6]. Note that in the bivariate case Hakopian's interpolation coincides with another (earlier) multivariate interpolation, considered by Cavaretta, Micchelli and Sharma [3]. In fact, the first method of recovering a polynomial of degree $n$ from its integrals over chords defined by equally spaced points $\left\{X_{i}\right\}$ on the boundary of the unit disk was described by Marr in [11]. Interpolation theorems based on integrals over chords can be used for approximate reconstruction of functions from their Radon transforms. Because of the importance of such recovery methods for applications in tomography, they have been intensively studied (see, for example, [11], [8], [10], [19], [4] and the bibliography therein).

Hakopian's interpolation theorem is the only result in the literature that describes a general configuration of chords which generates a poised (solvable) interpolation for any degree $n$ of the polynomial. The purpose of this paper is to provide another interpolation theorem, based on integrals over $(n+1)(n+2) / 2$ chords taken in $n+1$ distinct directions.

2. Preliminaries. We denote by $\Pi_{n}\left(\mathbb{R}^{2}\right)$ the set of all real algebraic polynomials in two variables of total degree $n$, that is,

$$
\Pi_{n}\left(\mathbb{R}^{2}\right):=\left\{\sum_{i+j \leq n} \alpha_{i j} x^{i} y^{j}: \alpha_{i j} \in \mathbb{R}\right\} .
$$

The set of univariate polynomials of degree $n$ will be designated by $\Pi_{n}$.

We shall consider real functions $f$ on the plane $\mathbb{R}^{2}$. The points of $\mathbb{R}^{2}$ will be denoted by $\mathbf{x}=(x, y)$. Also, we shall assume throughout this paper that the functions $f$ which are to be approximated have their support in the unit disk $\mathbf{B}:=\{\mathbf{x}:\|\mathbf{x}\| \leq 1\}$. Here, as usual, $\|\cdot\|$ is the Euclidean norm, $\|\mathbf{x}\|=\left(x^{2}+y^{2}\right)^{1 / 2}$.

It will be supposed that the integrals of $f$ are known along different lines intersecting the unit ball $\mathbf{B}$. Given $t \in[-1,1]$ and an angle $\theta \in[0, \pi)$, measured counterclockwise from the positive $x$-axis, we define the line $\ell$ by the equation

$$
\ell(x, y):=x \cos \theta+y \sin \theta-t=0 .
$$

The points $(x, y)$ on the finite segment $I(\theta, t):=\ell \cap \mathbf{B}$ can be represented as follows:

$$
x=t \cos \theta-s \sin \theta, \quad y=t \sin \theta+s \cos \theta
$$

for $s \in\left[-\sqrt{1-t^{2}}, \sqrt{1-t^{2}}\right]$.

For $t \in[-1,1]$ the Radon projection $\mathcal{R}_{\theta}(f ; t)$ of $f$ in direction $\theta$ is defined by 


$$
\begin{aligned}
\mathcal{R}_{\theta}(f ; t): & =\int_{I(\theta, t)} f(\mathbf{x}) d \mathbf{x} \\
& =\int_{-\sqrt{1-t^{2}}}^{\sqrt{1-t^{2}}} f(t \cos \theta-s \sin \theta, t \sin \theta+s \cos \theta) d s .
\end{aligned}
$$

In the literature it is also called an $X$-ray. Let us mention that

$$
\mathcal{R}_{\theta}(f ; t) \equiv \mathcal{R}_{\theta+\pi}(f ;-t) .
$$

Thus, two angles $\theta_{1}$ and $\theta_{2}$ which are equal mod $\pi$ produce the same projection. That is why we assume in what follows that $0 \leq \theta<\pi$.

The Radon transform

$$
f \mapsto\left\{\mathcal{R}_{\theta}(f ; t):-1 \leq t \leq 1,0 \leq \theta<\pi\right\}
$$

determines $f$ uniquely. This was proved by Radon [15] and John [9] for differentiable functions. A recent result of [19] (treating the general $d$-dimensional case) implies the following.

TheOREM A. If $f \in L^{1}\left(\mathbb{R}^{2}\right)$ has compact support in $\mathbf{B}$, then $f$ is uniquely determined by any infinite set of $X$-rays.

Since the function $f \equiv 0$ has all its projections equal to zero, the above theorem implies that the only function that has zero Radon transform is the zero function.

The Radon projection is easily computed in the special case when $f$ has a "plane wave" structure. Let us describe it more precisely. Let $\varrho(t)$ be a given univariate function and for a given angle $\theta$ define the unit vector $\xi=(\cos \theta, \sin \theta)$. The function

$$
\varrho(\mathbf{x} \cdot \xi):=\varrho(x \cos \theta+y \sin \theta)
$$

is called a ridge function in direction $\theta$ corresponding to $\varrho(t)$ (with profile $\varrho$ ).

Clearly, any ridge function with direction $\theta$ takes constant values on every line which is perpendicular to $\xi$. Therefore, for every ridge function $\varrho(\mathbf{x} \cdot \xi)$,

$$
\mathcal{R}_{\theta}(\varrho ; t)=2 \sqrt{1-t^{2}} \varrho(t) .
$$

The Radon projections of an algebraic polynomial $P \in \Pi_{n}\left(\mathbb{R}^{2}\right)$ in a given direction $\theta$ can be easily found:

Lemma 1. Let $\theta$ be fixed. For every polynomial $P \in \Pi_{n}\left(\mathbb{R}^{2}\right)$, there exists a univariate polynomial $p$ of degree $n$ such that

$$
\mathcal{R}_{\theta}(P ; t)=\sqrt{1-t^{2}} p(t), \quad-1 \leq t \leq 1 .
$$

This is a particular case of a known multivariate result (see, for example, [2, Lemma 4.1]). 
Every algebraic polynomial $P \in \Pi_{n}\left(\mathbb{R}^{2}\right)$ is uniquely determined by only a finite number of projections.

Theorem B. Let $\theta_{0}<\ldots<\theta_{n}$ be any given angles in $[0, \pi)$. Then the projections

$$
\mathcal{R}_{\theta_{k}}(P ; t), \quad-1 \leq t \leq 1, \quad k=0, \ldots, n,
$$

determine $P$ uniquely.

Proof. Since $P(x, y)=\sum_{i+j \leq n} a_{i j} x^{i} y^{j}$, we have

$$
\mathcal{R}_{\theta}(P ; t)=\sum_{i+j \leq n} a_{i j} \int_{-\sqrt{1-t^{2}}}^{\sqrt{1-t^{2}}}(t \cos \theta-s \sin \theta)^{i}(t \sin \theta+s \cos \theta)^{j} d s .
$$

From the condition $i+j \leq n$ it follows that the integrand is a trigonometric polynomial of $\theta$ of degree $n$ and it remains so after integration on $s$. Since $\mathcal{R}_{\theta+\pi}(P ; t)=\mathcal{R}_{\theta}(P ;-t)$ and $\left\{\mathcal{R}_{\theta_{k}}(P ; t)\right\}_{k=0}^{n}$ are given, we can suppose that $\mathcal{R}_{\theta}(P ; t)$ is known for $2 n+2$ different values of $\theta$ in $[0,2 \pi)$. Now, every trigonometric polynomial of degree $n$ is determined by its values at $2 n+1$ points in $[0,2 \pi)$. It follows that we know $\mathcal{R}_{\theta}(P ; t)$ for any $\theta$. Using the Radon theorem (Theorem A) we conclude that $f$ is uniquely determined by $\mathcal{R}_{\theta_{k}}(P ; t), k=0, \ldots, n$. The proof is complete.

In view of the remark after Theorem A, the following is an immediate consequence.

Corollary 1. Let $0 \leq \theta_{0}<\ldots<\theta_{n}<\pi$. If $P \in \Pi_{n}\left(\mathbb{R}^{2}\right)$ and $\mathcal{R}_{\theta_{k}}(P ; t)=0$ for $k=0, \ldots, n$, then $P \equiv 0$.

Our goal is to interpolate $f$ by algebraic polynomials from $\Pi_{n}\left(\mathbb{R}^{2}\right)$ using a finite number of integrals $\left\{\mathcal{R}_{\theta_{i}}\left(f ; t_{j}\right)\right\}$. Note that the dimension of the linear space $\Pi_{n}\left(\mathbb{R}^{2}\right)$ is equal to the number of the monomials $\left\{x^{i} y^{j}: i+j \leq n\right\}$ :

$$
\operatorname{dim} \Pi_{n}\left(\mathbb{R}^{2}\right)=d(n):=\left(\begin{array}{c}
n+2 \\
2
\end{array}\right) .
$$

When studying functions on $\mathbf{B}$ it is often helpful to use another basis in $\Pi_{n}\left(\mathbb{R}^{2}\right)$ which is generated by the Chebyshev polynomials of the second kind

$$
U_{m}(t):=\frac{1}{\sqrt{\pi}} \frac{\sin (m+1) \theta}{\sin \theta}, \quad t=\cos \theta
$$

It is well known (see, for example, [16]) that $U_{m}(t)$ is orthogonal to all polynomials of degree less than $m$ on $[-1,1]$ with respect to the weight function $\sqrt{1-t^{2}}$. The polynomials $\left\{U_{m}\right\}$ are normalized so that

$$
\int_{\mathbf{B}} U_{m}^{2}(y) d x d y=1
$$


For a given $\theta($ and $\xi:=(\cos \theta, \sin \theta))$, construct the ridge polynomial

$$
U_{m}(\theta ; \mathbf{x}):=U_{m}(\mathbf{x} \cdot \xi)=U_{m}(x \cos \theta+y \sin \theta),
$$

which takes the same value $U_{m}(t)$ at all points on the line at distance $t$ from the origin and making angle $\theta$ with the $x$-axis. According to Lemma 1 , the projection of $U_{m}(\theta ; \mathbf{x})$ at any direction $\varphi$ can be expressed through a univariate polynomial $p$ which is supposed to depend on $\varphi$. But the next lemma shows that it is actually the same for any $\varphi$, and moreover, it is just $U_{m}(t)$.

Lemma 2. For each $t \in(-1,1), \theta$ and $\varphi$, we have

$$
\mathcal{R}_{\varphi}\left(U_{m}(\theta ; \cdot) ; t\right)=\frac{2}{m+1} \sqrt{1-t^{2}} U_{m}(t) \frac{\sin (m+1)(\varphi-\theta)}{\sin (\varphi-\theta)} .
$$

This remarkable relation was established by Marr in [11]. We shall call it Marr's formula. Different proofs have been given by many authors (see, for example [1, Corollary 2]). Marr's formula is a key equality for the Chebyshev-Fourier analysis on $\mathbf{B}$. It shows the way to reduce the multivariate case to a univariate one. The following useful relation can be easily derived from Marr's formula.

Corollary 2. For any two angles $\theta$ and $\varphi$ we have

$$
\int_{\mathbf{B}} U_{m}(\theta ; \mathbf{x}) U_{m}(\varphi ; \mathbf{x}) d \mathbf{x}=\frac{1}{m+1} \frac{\sin (m+1)(\varphi-\theta)}{\sin (\varphi-\theta)} .
$$

Proof. Using the fact that $U_{m}(\varphi ; \mathbf{x})=U_{m}(t)$ for every $\mathbf{x}$ on the line segment $I(\varphi, t)$, we get

$$
\begin{aligned}
\int_{\mathbf{B}} U_{m}(\theta ; \mathbf{x}) U_{m}(\varphi ; \mathbf{x}) d \mathbf{x} & =\int_{-1}^{1}\left[\int_{I(\varphi, t)} U_{m}(\theta ; \mathbf{x}) U_{m}(\varphi ; \mathbf{x}) d \mathbf{x}\right] d t \\
& =\int_{-1}^{1} U_{m}(t) \mathcal{R}_{\varphi}\left(U_{m}(\theta ; \cdot) ; t\right) d t .
\end{aligned}
$$

Then substituting the expression from Lemma 2 for $\mathcal{R}_{\varphi}\left(U_{m}(\theta ; \cdot) ; t\right)$, and taking into account the normalization $\int_{-1}^{1} \sqrt{1-t^{2}} U_{m}^{2}(t) d t=1 / 2$, we obtain the result.

Similarly one can see that for every $\theta$ the ridge polynomial $U_{m}(\theta ; t)$ is orthogonal to every polynomial from $\Pi_{m-1}\left(\mathbb{R}^{2}\right)$ on $\mathbf{B}$, i.e.,

$$
\int_{\mathbf{B}} U_{m}(\theta ; \mathbf{x}) P(\mathbf{x}) d \mathbf{x}=0 \quad \text { for every } P \in \Pi_{m-1}\left(\mathbb{R}^{2}\right) .
$$


Indeed, making use of Lemma 1, we obtain

$$
\begin{aligned}
\int_{\mathbf{B}} U_{m}(\theta ; \mathbf{x}) P(\mathbf{x}) d \mathbf{x} & =\int_{-1}^{1} U_{m}(t) \mathcal{R}_{\theta}(P ; t) d t \\
& =\int_{-1}^{1} \sqrt{1-t^{2}} U_{m}(t) p(t) d t=0 .
\end{aligned}
$$

The ridge polynomials $U_{m}(\theta ; t)$ with equally spaced angles $\theta$ play a fundamental role in the space of bivariate polynomials, as can be seen from the following proposition. It is an immediate consequence of (1) and Corollary 2.

Theorem C. Let

and

$$
\theta_{m j}:=\frac{j \pi}{m+1}, \quad m \in \mathbb{N}, j=0, \ldots, m,
$$

$$
U_{m j}(\mathbf{x}):=U_{m}\left(\theta_{m j} ; \mathbf{x}\right) .
$$

The ridge polynomials $\left\{U_{m j}\right\}$ form an orthonormal basis in $\Pi_{n}\left(\mathbb{R}^{2}\right)$ on $\mathbf{B}$.

More about the polynomials $\left\{U_{m j}\right\}$ and other systems of orthogonal polynomials in $d$ variables can be found in [5] and [14].

3. The interpolation theorem. By Theorem B, every polynomial $P \in \Pi_{n}\left(\mathbb{R}^{2}\right)$ is uniquely determined by its Radon projections in $n+1$ distinct directions $\theta_{0}, \ldots, \theta_{n}$. In other words, given the functions

$$
\mathcal{R}_{\theta_{0}}(P ; t), \ldots, \mathcal{R}_{\theta_{n}}(P ; t)
$$

and the information that they are Radon projections of an algebraic polynomial $P$ of degree less than or equal to $n$, one can identify the polynomial $P$ uniquely. According to Lemma 1,

$$
\mathcal{R}_{\theta}(P ; t)=\sqrt{1-t^{2}} p(t)
$$

with some $p \in \Pi_{n}$. Therefore, we do not need to know the values of $\mathcal{R}_{\theta}(P ; t)$ for all $t$. It would be enough to compute the Radon projection $\mathcal{R}_{\theta}(P ; t)$ for $n+1$ distinct values of $t$ only. Then the value of $\mathcal{R}_{\theta}(P ; t)$ can be recovered at any point $t$ by Lagrange interpolation. Thus we face the following problem: Suppose we know the values

$$
\mathcal{R}_{\theta_{i}}\left(P ; t_{i j}\right), \quad i=0, \ldots, n, j=0, \ldots, n,
$$

for some $0 \leq \theta_{0}<\ldots<\theta_{n}<\pi$ and $-1<t_{i 0}<\ldots<t_{i n}<1, i=0, \ldots, n$. How to reconstruct $P$, provided $P \in \Pi_{n}\left(\mathbb{R}^{2}\right)$ ?

On account of Theorem $\mathrm{C}$ we can write $P$ in the form

$$
P(\mathbf{x})=\sum_{m=0}^{n} \sum_{j=0}^{m} a_{m j}(P) U_{m j}(\mathbf{x})
$$


with

$$
a_{m j}(P)=\int_{\mathbf{B}} P(\mathbf{x}) U_{m j}(\mathbf{x}) d x .
$$

Our goal is to find the coefficients $a_{m j}$. If we choose $t_{i 0}, \ldots, t_{i n}$ to be the zeros $\eta_{0}, \ldots, \eta_{n}$ of $U_{n+1}(t)$, then we can easily calculate $a_{m j}$, and hence find $P$ using the Gaussian cubature formula

$$
\int_{\mathbf{B}} f(\mathbf{x}) d \mathbf{x} \approx \sum_{k=0}^{n} A_{k} \int_{I_{k}} f
$$

which was found in [1]. Here $\int_{I_{k}}$ denotes the line integral over the chord $I_{k}$ passing through $x=\eta_{k}$ and parallel to the $y$-axis. The coefficients are explicitly given in [1]. The cubature is exact for every polynomial $f \in \Pi_{2 n+1}\left(\mathbb{R}^{2}\right)$ and is unique (up to rotation) with the highest degree of precision (see [2]). Since deg $P U_{m j} \leq 2 n$, we can compute the integral for $a_{m j}(P)$ exactly by an appropriate rotation of $(3)$ (so that the $x$-axis goes to direction $\theta_{m j}$ ) using the values

$$
\int_{I_{k}} P U_{m j}=U_{m}\left(\eta_{k}\right) \mathcal{R}_{\theta_{m j}}\left(P ; \eta_{k}\right), \quad k=0, \ldots, n .
$$

Note that $\mathcal{R}_{\theta_{m j}}\left(P ; \eta_{k}\right)$ can be found via trigonometric interpolation since $\mathcal{R}_{\theta}\left(P ; \eta_{k}\right)$ is given for $n+1$ values of $\theta$ (namely, for $\left.\theta=\theta_{0}, \ldots, \theta_{n}\right)$. Therefore, the cubature formula (3) allows us to reconstruct every polynomial $P \in \Pi_{n}\left(\mathbb{R}^{2}\right)$ from $(n+1)^{2}$ Radon projections. But $(n+1)^{2}$ is twice the dimension $d(n)$ of $\Pi_{n}\left(\mathbb{R}^{2}\right)$. Is there a way to recover $P$ using exactly $d(n)$ values? In other words, is there a configuration of $d(n)$ chords $\left\{I\left(\theta_{i}, t_{j}\right)\right\}_{(i, j) \in J}$ $(|J|=d(n))$ in the disk $\mathbf{B}$ such that the interpolation problem

$$
\int_{I\left(\theta_{i}, t_{j}\right)} P(\mathbf{x}) d \mathbf{x}=\gamma_{i j}, \quad(i, j) \in J, P \in \Pi_{n}\left(\mathbb{R}^{2}\right),
$$

is poised (i.e., has a unique solution for any given data $\left.\left\{\gamma_{i j}\right\}\right)$ ? As already mentioned in the introduction, Hakopian's result [6] says that if $I\left(\theta_{i}, t_{j}\right)$ are the linear segments joining any 2 points from any preassigned set of $n+2$ points on the boundary $\partial \mathbf{B}$ of $\mathbf{B}$, then the corresponding interpolation problem is poised. This is the only known non-trivial configuration of chords for poised interpolation that works for every $n$. Note that in the Hakopian case one needs to compute the Radon projections in $d(n)$ different directions $\theta_{i}$. Such data come from measuring $X$-rays issuing from $n+1$ distinct points on $\partial \mathbf{B}$. Another situation of practical importance is when the $X$-rays are taken in $n+1$ groups, all rays in each group being parallel. This is the situation we are considering. It can be described by a set of given angles $\theta_{0}<\ldots<\theta_{n}$ in $[0, \pi)$ and a triangular matrix $T=\left\{t_{k i}\right\}$ of points

$$
t_{k k}<\ldots<t_{k n}, \quad k=0, \ldots, n,
$$


associated with the angles. The problem is to characterize all locations of the nodes $\left\{t_{k j}\right\}$ for which the interpolation of the set of data $\left\{\mathcal{R}_{\theta_{k}}\left(\cdot ; t_{k j}\right)\right\}$ by polynomials of degree $n$ is poised. For example, in case $n=1$, the set of data consists of three integrals: one over a chord in direction $\theta_{0}$ and two others, parallel, in direction $\theta_{1}$. It can be easily seen in this simple case that the problem is poised if and only if $t_{00} \neq 0$. This can be written as $U_{1}\left(t_{00}\right) \neq 0$.

The matrices

$$
\mathbf{U}_{k}:=\left(\begin{array}{cccc}
U_{k}\left(t_{k k}\right) & U_{k+1}\left(t_{k k}\right) & \ldots & U_{n}\left(t_{k k}\right) \\
U_{k}\left(t_{k, k+1}\right) & U_{k+1}\left(t_{k, k+1}\right) & \ldots & U_{n}\left(t_{k, k+1}\right) \\
\ldots \ldots \ldots \ldots \ldots \ldots \ldots \ldots \ldots \ldots & \ldots \ldots \ldots \ldots \\
U_{k}\left(t_{k n}\right) & U_{k+1}\left(t_{k n}\right) & \ldots & U_{n}\left(t_{k n}\right)
\end{array}\right)
$$

play a crucial role in the interpolation problem we consider here.

TheOREM 1. For given angles $0 \leq \theta_{0}<\ldots<\theta_{n}<\pi$ and associated points $T=\left\{t_{k i}\right\}_{k=0, i=k}^{n}$, the interpolation problem

$$
\int_{I\left(\theta_{k}, t_{k i}\right)} P(\mathbf{x}) d \mathbf{x}=\gamma_{k i}, \quad k=0, \ldots, n, i=k, \ldots, n, P \in \Pi_{n}\left(\mathbb{R}^{2}\right),
$$

is poised if and only if

$$
\operatorname{det} \mathbf{U}_{k} \neq 0 \quad \text { for } k=0, \ldots, n \text {. }
$$

Proof. By Lemma 1, for each $k$ we can write

$$
\mathcal{R}_{\theta_{k}}(P ; t)=\sqrt{1-t^{2}} p_{k}(t)
$$

with some univariate polynomial $p_{k}$ of degree $n$. Expanding now $p_{k}$ in a Chebyshev series, we obtain

where

$$
\mathcal{R}_{\theta_{k}}(P ; t)=\sqrt{1-t^{2}} \sum_{i=0}^{n} b_{k i}(P) U_{i}(t),
$$

$$
b_{k i}(P)=2 \int_{-1}^{1} \mathcal{R}_{\theta_{k}}(P ; t) U_{i}(t) d t=2 \int_{\mathbf{B}} P(\mathbf{x}) U_{i}\left(\theta_{k} ; \mathbf{x}\right) d \mathbf{x} .
$$

On the other hand, using (2), we can compute $\mathcal{R}_{\theta_{k}}(P ; t)$ in terms of $\left\{a_{m j}(P)\right\}$. Indeed, by Marr's formula,

$$
\begin{aligned}
\mathcal{R}_{\theta_{k}}(P ; t) & =\sum_{m=0}^{n} \sum_{j=0}^{m} a_{m j} \mathcal{R}_{\theta_{k}}\left(U_{m j} ; t\right) \\
& =\sum_{m=0}^{n} \sum_{j=0}^{m} a_{m j} \frac{2}{m+1} \sqrt{1-t^{2}} U_{m}(t) \frac{\sin (m+1)\left(\theta_{m j}-\theta_{k}\right)}{\sin \left(\theta_{m j}-\theta_{k}\right)} \\
& =\sqrt{1-t^{2}} \sum_{m=0}^{n}\left(\sum_{j=0}^{m} s_{m k j} a_{m j}\right) U_{m}(t)
\end{aligned}
$$


where we have used the notation

$$
s_{m k j}:=\frac{2}{m+1} \frac{\sin (m+1)\left(\theta_{m j}-\theta_{k}\right)}{\sin \left(\theta_{m j}-\theta_{k}\right)} .
$$

The last two representations of $\mathcal{R}_{\theta_{k}}(P ; t)$ lead to the equality

$$
\sum_{m=0}^{n}\left(\sum_{j=0}^{m} s_{m k j} a_{m j}\right) U_{m}(t)=\sum_{i=0}^{n} b_{k i} U_{i}(t) .
$$

Comparing the coefficients of $U_{m}(t)$ on both sides of (5) we arrive at the relation $s_{m k 0} a_{m 0}+\ldots+s_{m k m} a_{m m}=b_{k m}$. It was derived for any particular direction $\theta_{k}$. Writing it for $k=0, \ldots, m$, we obtain the system

$$
\begin{gathered}
s_{m 00} a_{m 0}+\ldots+s_{m 0 m} a_{m m}=b_{0 m}, \\
\vdots \\
s_{m m 0} a_{m 0}+\ldots+s_{m m m} a_{m m}=b_{m m} .
\end{gathered}
$$

Consider the matrix $\mathbf{S}:=\left\{s_{m k j}\right\}$ of this system. We shall show that $\operatorname{det} \mathbf{S} \neq 0$. Note that $\mathbf{S}=\left\{D_{m}\left(\theta_{m j}-\theta_{k}\right)\right\}_{k=0, j=0}^{m}$ where

$$
D_{m}(\theta):=\frac{2}{m+1} \frac{\sin (m+1) \theta}{\sin \theta}
$$

is the Dirichlet kernel. The fact that $\operatorname{det}\left\{D_{m}\left(\theta_{j}-\theta_{k}\right)\right\}_{k=0, j=0}^{m} \underset{m}{m}$ is non-zero is known, i.e., in case $\left\{\theta_{k}\right\} \equiv\left\{\theta_{m j}\right\}$ the claim is true. We need it for any $\left\{\theta_{k}\right\}$. Our proof relies on the fact that the polynomials

$$
U_{m}\left(\theta_{0} ; \mathbf{x}\right), \ldots, U_{m}\left(\theta_{m} ; \mathbf{x}\right)
$$

are linearly independent for every $0 \leq \theta_{0}<\ldots<\theta_{m}<\pi$. This property was mentioned in [8] and follows from Lemma 3.2 there. For the sake of completeness, we sketch the proof here.

Assume that

$$
u(\mathbf{x}):=\alpha_{0} U_{m}\left(\theta_{0} ; \mathbf{x}\right)+\ldots+\alpha_{m} U_{m}\left(\theta_{m} ; \mathbf{x}\right) \equiv 0 \quad \text { on } \mathbf{B} .
$$

Then

$$
\begin{aligned}
0 & =\left(\frac{\partial}{\partial x}\right)^{i}\left(\frac{\partial}{\partial y}\right)^{m-i} u(\mathbf{x}) \\
& =\sum_{k=0}^{m} \alpha_{k} U_{m}^{(m)}\left(x \cos \theta_{k}+y \sin \theta_{k}\right)\left(\cos \theta_{k}\right)^{i}\left(\sin \theta_{k}\right)^{m-i}
\end{aligned}
$$

At $\mathbf{x}=0$ we obtain

$$
0=U_{m}^{(m)}(0) \sum_{k=0}^{m}\left(\cos \theta_{k}\right)^{i}\left(\sin \theta_{k}\right)^{m-i} \alpha_{k} .
$$


Considering this equation for $i=0, \ldots, m$, we derive a linear system with respect to $\alpha_{0}, \ldots, \alpha_{m}$. If $\theta_{0} \neq 0$ the matrix of the system is (up to a non-zero constant multiplier)

$$
D_{m}\left(\theta_{0}, \ldots, \theta_{m}\right):=\left(\begin{array}{cccc}
1 & 1 & \ldots & 1 \\
\cot \theta_{0} & \cot \theta_{1} & \ldots & \cot \theta_{m} \\
\ldots \ldots & \ldots & \ldots & \ldots \\
\ldots & \ldots & \ldots & \ldots \\
\left(\cot \theta_{0}\right)^{m} & \left(\cot \theta_{1}\right)^{m} & \ldots & \left(\cot \theta_{m}\right)^{m}
\end{array}\right)
$$

while in case $\theta_{0}=0$ it is $D_{m-1}\left(\theta_{1}, \ldots, \theta_{m}\right)$. In both cases it is a Vandermonde matrix, and thus non-singular since $\cot \theta_{i} \neq \cot \theta_{j}$ if $i \neq j$. Therefore $\alpha_{0}=$ $\ldots=\alpha_{m}=0$ and the linear independence of $U_{m}\left(\theta_{0} ; \mathbf{x}\right), \ldots, U_{m}\left(\theta_{m} ; \mathbf{x}\right)$ is proved.

Now let us return to the proof of non-singularity of $\mathbf{S}$. Assume that $\operatorname{det} \mathbf{S}=0$. Then there exist numbers $c_{0}, \ldots, c_{m}$, not all zero, such that

$$
\sum_{k=0}^{m} c_{k} s_{m k j}=0 \quad \text { for } j=0, \ldots, m .
$$

By Corollary 2,

$$
s_{m k j}=\left(U_{m}\left(\theta_{k} ; \cdot\right), U_{m j}\right):=\int_{\mathbf{B}} U_{m}\left(\theta_{k} ; \mathbf{x}\right) U_{m j}(\mathbf{x}) d \mathbf{x} .
$$

Thus

$$
\int_{\mathbf{B}}\left(\sum_{k=0}^{m} c_{k} U_{m}\left(\theta_{k} ; \mathbf{x}\right)\right) U_{m j}(\mathbf{x}) d \mathbf{x}=0, \quad j=0, \ldots, m,
$$

and therefore the polynomial

$$
Q(\mathbf{x}):=\sum_{k=0}^{m} c_{k} U_{m}\left(\theta_{k} ; \mathbf{x}\right)
$$

is orthogonal to $U_{m j}$ for $j=0, \ldots, m$. In view of (1), $Q$ is also orthogonal to $U_{i j}$ for $i \leq m-1$ and $j=0, \ldots, i$. Thus, by Theorem C, $Q(\mathbf{x}) \equiv 0$. Now using the fact that $\left\{U_{m}\left(\theta_{k} ; \mathbf{x}\right)\right\}_{k=0}^{m}$ are linearly independent, we conclude that $c_{0}=\ldots=c_{m}=0$, a contradiction. Therefore $\operatorname{det} \mathbf{S} \neq 0$. Consequently, given $b_{0 m}, \ldots, b_{m m}$ we can compute the coefficients $a_{m 0}, \ldots, a_{m m}$ by solving the linear system (6).

We have just proved the following auxiliary proposition:

Given any numbers $\left\{\beta_{m j}\right\}_{m=0, j=m}^{n}$, there exists a unique polynomial $P \in$ $\Pi_{n}\left(\mathbb{R}^{2}\right)$ such that

$$
b_{m j}(P)=\beta_{m j}, \quad m=0, \ldots, n, j=m, \ldots, n .
$$

The next task is to show that any of the functionals $\left\{b_{m j}(P)\right\}$ can be com- 
puted from $d(n)$ Radon projection beams

$$
\mathcal{A}:=\left\{\mathcal{R}_{\theta_{k}}\left(P ; t_{k j}\right)\right\}_{k=0, j=k}^{n} \underset{n}{ } .
$$

For a fixed $(m, j)$ the quantity $b_{m j}(P)$ is a linear additive, homogeneous functional on $\Pi_{n}\left(\mathbb{R}^{2}\right)$. The set $\mathcal{A}$ consists of $d(n)$ such functionals. Thus, there exists a formula of the form

$$
b_{m j}(P)=\sum_{k=0}^{n} \sum_{i=k}^{n} C_{k i} \mathcal{R}_{\theta_{k}}\left(P ; t_{k i}\right), \quad \forall P \in \Pi_{n}\left(\mathbb{R}^{2}\right),
$$

if and only if

$$
\mathcal{R}_{\theta_{k}}\left(P ; t_{k i}\right)=0, k=0, \ldots, n, i=k, \ldots, n \Rightarrow b_{m j}(P)=0 .
$$

This is a simple observation based on linear algebra arguments. A more general statement for any number $N$ of functionals (including $N \leq d(n)$ ) is due to Sobolev [18] (see also [12, Theorem 3.5]).

Assume that the left hand side of (7) holds. By an induction argument, we shall derive the conclusion on the right side of ( 7 ) for every $m=0, \ldots, n$, $j=0, \ldots, n$.

Let $k=0$. By assumption, we have

$$
\mathcal{R}_{\theta_{0}}\left(P ; t_{00}\right)=\ldots=\mathcal{R}_{\theta_{0}}\left(P ; t_{0 n}\right)=0 .
$$

In view of Lemma 1 , this implies $\mathcal{R}_{\theta_{0}}(P ; t) \equiv 0$. Then

$$
\sum_{i=0}^{n} b_{0 i}(P) U_{i}(t) \equiv 0
$$

and hence, because of the linear independence of $\left\{U_{i}\right\}$,

$$
b_{0 i}(P)=0 \quad \text { for } i=0, \ldots, n .
$$

The first step of the induction procedure is complete. Note that the relation $a_{00}(P)=\frac{1}{2} b_{00}(P)$ implies $a_{00}(P)=0$. Therefore, in the first step we have shown that $P$ is of the form

$$
P(\mathbf{x})=a_{10} U_{10}(\mathbf{x})+a_{11} U_{11}(\mathbf{x})+\ldots+a_{n n} U_{n n}(\mathbf{x}) .
$$

Assume now that after $k$ steps we have proved that

$$
b_{i, j}(P)=0 \quad \text { for } i=0, \ldots, k-1, j=0, \ldots, n,
$$

and $P$ reduces to

$$
P(\mathbf{x})=\sum_{i=k}^{n} \sum_{j=0}^{i} a_{i j} U_{i j}(\mathbf{x}) .
$$

Then, by Marr's formula, the Radon projection of $P$ in direction $\theta_{k}$ will take the form

$$
\mathcal{R}_{\theta_{k}}(P ; t)=\sqrt{1-t^{2}}\left(b_{k k} U_{k}(t)+\ldots+b_{k n} U_{n}(t)\right)
$$


which shows that $b_{k 0}=\ldots=b_{k, k-1}=0$. To prove that the remaining coefficients are also zero we use the assumptions

$$
\mathcal{R}_{\theta_{k}}\left(P ; t_{k k}\right)=\ldots=\mathcal{R}_{\theta_{k}}\left(P ; t_{k n}\right)=0 .
$$

They produce a system of linear equations

$$
b_{k k} U_{k}\left(t_{k j}\right)+\ldots+b_{k n} U_{n}\left(t_{k j}\right)=0, \quad j=k, \ldots, n,
$$

which has a unique solution if and only if $\operatorname{det} \mathbf{U}_{k} \neq 0$. Thus, assuming the latter, we conclude that

$$
b_{k j}(P)=0 \quad \text { for } j=k, \ldots, n,
$$

and therefore, for all $j=0, \ldots, n$. Now it follows from (6) that $a_{k 0}=\ldots=$ $a_{k k}=0$, and therefore

$$
P(\mathbf{x})=\sum_{i=k+1}^{n} \sum_{j=0}^{i} a_{i j} U_{i j}(\mathbf{x}) .
$$

The induction step is complete. After $n+1$ steps we get $P(\mathbf{x}) \equiv 0$. The theorem is proved.

REMARK. The referee suggested a more transparent proof of the necessity part of Theorem 1 . Let us sketch his/her idea.

Suppose that $\operatorname{det} \mathbf{U}_{\mu}=0$ for some $0 \leq \mu \leq n$. Choose the maximal such $\mu$. Thus $\operatorname{det} \mathbf{U}_{k} \neq 0$ for all $k>\mu$. We shall show that there is a non-zero polynomial $P \in \Pi_{n}\left(\mathbb{R}^{2}\right)$ whose interpolation parameters are all zero. This will be done by choosing a corresponding (nonzero) matrix

$$
\mathcal{B}(P)=\left\{b_{k j}(P)\right\}_{k=0, j=0}^{n} .
$$

Note that by the auxiliary proposition given above (in the proof of Theorem 1) all components of $\mathcal{B}(P)$ are uniquely determined by the components on and above the diagonal. Moreover, it can be shown that the first $m$ columns of $\mathcal{B}(P)$ (for any $m$ ) are determined by their components lying on and above the diagonal. Let us choose the first $\mu$ rows of the matrix $\mathcal{B}(P)$ (i.e., the rows with indices $k=0, \ldots, \mu-1$ ) to be zero. Then, by the observation above, also the first $\mu$ columns will be identically 0 . Next, in view of the assumption $\operatorname{det} \mathbf{U}_{\mu}=0$, the remaining $n-\mu+1$ elements of the $\mu$ th row can be found as a non-zero solution of a homogeneous singular system induced by the conditions

$$
\mathcal{R}_{\theta_{\mu}}\left(P, t_{\mu \mu}\right)=\ldots=\mathcal{R}_{\theta_{\mu}}\left(P, t_{\mu n}\right)=0 .
$$

Thus, we have chosen $\mu$ rows and therefore the $\mu$ th column is determined. Then in the $(\mu+1)$ th row the remaining elements will be uniquely determined from the corresponding non-singular and (possibly) non-homogeneous system. And so on. 
The next observation follows as a particular case.

TheOrem 2. Let $0 \leq \theta_{0}<\ldots<\theta_{n}<\pi$ be any given angles and let $T$ be a set of arbitrary $n+1$ distinct points in $(-1,1)$. Then there exists a numbering $t_{0}, \ldots, t_{n}$ of the points from $T$ so that the interpolation problem

$$
\int_{I\left(\theta_{i}, t_{j}\right)} P(\mathbf{x}) d \mathbf{x}=\gamma_{i j}, \quad i=0, \ldots, n, j=i, \ldots, n,
$$

is poised in $\Pi_{n}\left(\mathbb{R}^{2}\right)$.

Proof. For any set $T$ of distinct points $\left\{t_{j}\right\}$ the matrix

$$
\mathbf{U}_{0}:=\left\{U_{m}\left(t_{j}\right)\right\}_{j=0, m=0}^{n} \underset{n}{n}
$$

is non-singular. Then at least one of its minors, obtained from $\mathbf{U}_{0}$ by deleting the first column and one row, is non-zero. We let $t_{0}$ be the point from $T$, corresponding to this row. We next determine $t_{1}$ so that the minor obtained from $\mathbf{U}_{0}$ by deleting the first two columns and the rows corresponding to $t_{0}$ and $t_{1}$ be non-zero, and so on until we order all the points of $T$ in a sequence $t_{0}, \ldots, t_{n}$ such that

$$
\operatorname{det} \mathbf{U}_{k}:=\left(\begin{array}{cccc}
U_{k}\left(t_{k}\right) & U_{k+1}\left(t_{k}\right) & \ldots & U_{n}\left(t_{k}\right) \\
U_{k}\left(t_{k+1}\right) & U_{k+1}\left(t_{k+1}\right) & \ldots & U_{n}\left(t_{k+1}\right) \\
\ldots \ldots \ldots \ldots \ldots \ldots \ldots \ldots \ldots \ldots \ldots \ldots & \ldots \ldots \ldots \\
U_{k}\left(t_{n}\right) & U_{k+1}\left(t_{n}\right) & \ldots & U_{n}\left(t_{n}\right)
\end{array}\right) \neq 0
$$

for $k=0, \ldots, n$. Then we apply Theorem 1 .

4. A remark on inversion of the Radon transform. Assume that we know a priori that the function $f$ can be represented as a uniformly convergent series on $\mathbf{B}$ of the form

$$
f(\mathbf{x})=\sum_{m=0}^{\infty} \sum_{j=0}^{m} a_{m j}(f) U_{m j}(\mathbf{x}) .
$$

Then the partial sum

$$
S_{n}(f ; \mathbf{x}):=\sum_{m=0}^{n} \sum_{j=0}^{m} a_{m j}(f) U_{m j}(\mathbf{x})
$$

can be used as an approximation of $f(\mathbf{x})$. Suppose that every Radon projection $\mathcal{R}_{\theta}(f ; t)$ is known or easily available. The results of the previous section suggest a way to recover $S_{n}(f ; \mathbf{x})$ from $\left\{\mathcal{R}_{\theta}(f ; t)\right\}$ for every $n$, and thus, to describe an inversion of the Radon transform. In order to do this, assume 
that every $\mathcal{R}_{\theta}(f ; t)$ is given as a Chebyshev-Fourier series

$$
\mathcal{R}_{\theta}(f ; t)=\sqrt{1-t^{2}}\left(\sum_{m=0}^{\infty} b_{m}(f ; \theta) U_{m}(t)\right) .
$$

The coefficients $b_{m}(f ; \theta)$ can be easily computed from $\left\{\mathcal{R}_{\theta}(f ; t)\right\}$. Note that

$$
b_{m}(f ; \theta) \text { is a trigonometric polynomial of degree } m \text {. }
$$

To see this we apply $\mathcal{R}_{\theta}$ to $(8)$, use Marr's formula for $\mathcal{R}_{\theta}\left(U_{m j} ; t\right)$, and arrive, as in the derivation of (5), at the equality

$$
\sum_{j=0}^{m} a_{m j}(f) \frac{2}{m+1} \frac{\sin (m+1)\left(\theta_{m j}-\theta\right)}{\sin \left(\theta_{m j}-\theta\right)}=b_{m}(f ; \theta) .
$$

Now (9) is evident since $\frac{\sin (m+1) \varphi}{\sin \varphi}$ is a trigonometric polynomial of degree $m$.

INVERSION FORMULA. If the series (8) is uniformly convergent on $\mathbf{B}$, then

$$
f(\mathbf{x})=\frac{1}{2} \sum_{m=0}^{\infty} \sum_{j=0}^{m} b_{m}\left(f ; \theta_{m j}\right) U_{m j}(\mathbf{x})
$$

and, equivalently,

$$
f(\mathbf{x})=\frac{1}{4 \pi} \sum_{m=0}^{\infty}(m+1) \int_{0}^{2 \pi} b_{m}(f ; \theta) U_{m}(\theta ; \mathbf{x}) d \theta,
$$

for every $\mathbf{x} \in \mathbf{B}$.

To verify the first formula we need to show that $a_{m j}=\frac{1}{2} b_{m}\left(f ; \theta_{m j}\right)$. This follows from (10) or directly:

$$
b_{m}\left(f ; \theta_{m j}\right)=2 \int_{-1}^{1} \mathcal{R}_{\theta_{m j}}(f ; t) U_{m}(t) d t=2 \int_{\mathbf{B}} f(\mathbf{x}) U_{m j}(\mathbf{x}) d \mathbf{x}=2 a_{m j}(f) .
$$

The last relation can be used to find the coefficients $a_{m j}(f)$ from an infinite number of projections in directions $\left\{\theta_{m j}\right\}$. Indeed, according to $(9), b_{m}(f ; \theta)$ is a trigonometric polynomial of degree $m$. Hence it can be recovered from any $2 m+1$ values through Lagrange interpolation. Thus, we first compute the integral

$$
b_{m}(f ; \theta)=2 \int_{-1}^{1} \mathcal{R}_{\theta}(f ; t) U_{m}(t) d t
$$

for any $m+1$ values of $\theta, 0 \leq \theta<\pi$, next find also $b_{m}(f ; \theta+\pi)$ from the relation $\mathcal{R}_{\theta}(f ; t) \equiv \mathcal{R}_{\theta+\pi}(f ;-t)$, and then, by interpolation, calculate $b_{m}\left(f ; \theta_{m j}\right)$, and hence $a_{m j}(f)$. 
To derive the second formula for $f(\mathbf{x})$, we use the fact that the quadrature formula

$$
\int_{0}^{2 \pi} g(\theta) d \theta \approx \frac{2 \pi}{N+1} \sum_{j=0}^{N} g\left(\frac{2 \pi j}{N+1}\right)
$$

integrates exactly all trigonometric polynomials of degree not exceeding $N$. In view of this, taking into account the relations

$$
\begin{aligned}
b_{m}(f ; \theta+\pi) & =(-1)^{m} b_{m}(f ; \theta), \\
U_{m}(-t) & =(-1)^{m} U_{m}(t),
\end{aligned}
$$

and the notation

$$
\tau_{j}:=\frac{j \pi}{m+1}, \quad j=0, \ldots, 2 m+1,
$$

we obtain

$$
\begin{aligned}
\sum_{j=0}^{m} b_{m}\left(f ; \theta_{m j}\right) U_{m}\left(\theta_{m j} ; \mathbf{x}\right) & =\frac{1}{2} \sum_{j=0}^{2 m+1} b_{m}\left(f ; \tau_{j}\right) U_{m}\left(\tau_{j} ; \mathbf{x}\right) \\
& =\frac{m+1}{2 \pi} \int_{0}^{2 \pi} b_{m}(f ; \theta) U_{m}(\theta ; \mathbf{x}) d \theta
\end{aligned}
$$

and (11) is proven.

Note that the proof of (11) (in the $d$-dimensional case) that was given in [14] uses essentially the Radon inversion formula.

Another useful observation is that to any integrable function $f$ on $\mathbf{B}$ we can assign the sequence $\left\{b_{m}(f ; \theta)\right\}$ of trigonometric polynomials. This sequence determines $f$ uniquely. In case $f$ is an algebraic polynomial from $\Pi_{n}\left(\mathbb{R}^{2}\right)$, the sequence $\left\{b_{m}(f ; \theta)\right\}$ is finite, and consists of $n+1$ trigonometric polynomials. Moreover, any such sequence of $n+1$ polynomials $\left(b_{m}\right.$ being of degree $m$ ) determines uniquely an algebraic polynomial $f \in \Pi_{n}\left(\mathbb{R}^{2}\right)$. This one-to-one correspondence can be used to study $f$ in terms of the properties of the associated trigonometric polynomials. Actually, the notes in the present and in the next section are a result of this relation.

5. A remark on the Schinzel representation. Vostretsov and Kreines [20] proved the following representation result:

For any given set of $n+1$ non-co-linear unit vectors $\xi_{j}$ and a polynomial $P \in \Pi_{n}\left(\mathbb{R}^{2}\right)$, there exist univariate polynomials $r_{0}, \ldots, r_{n}$ of degree $n$ such that

$$
P(\mathbf{x})=\sum_{j=0}^{n} r_{j}\left(\mathbf{x} \cdot \xi_{j}\right)
$$


We shall call it the Vostretsov-Kreines lemma. A similar representation with an unspecified number of ridge functions was also given for polynomials of any dimension $d$. Recently Schinzel [17] gave an extension and refinement of the Vostretsov-Krelnes lemma which implies that for every given $P \in$ $\Pi_{n}\left(\mathbb{R}^{2}\right)$ one can reduce the number of ridge functions $\left\{r_{j}\right\}$ in (12) by 1 choosing the directions $\xi_{1}, \ldots, \xi_{n}$ appropriately. Moreover, $n$ is the minimal number with this property (see also the examples in Oskolkov [13]).

Following the idea of proof of our interpolation theorem we first give a simple constructive proof of the Vostretsov-Krelnes lemma and then, using it, we refine Schinzel's result for polynomials $P \in \Pi_{n}\left(\mathbb{R}^{2}\right)$ of odd degree.

REPRESEnTATion Formula. Let $\theta_{0}, \ldots, \theta_{n}$ be any set of fixed distinct angles in $[0, \pi)$. Then every polynomial $P \in \Pi_{n}\left(\mathbb{R}^{2}\right)$ can be uniquely represented in the form

$$
P(\mathbf{x})=\sum_{j=0}^{n} \sum_{m=j}^{n} c_{m j} U_{m}\left(\mathbf{x} \cdot \xi_{j}\right), \quad \xi_{j}:=\left(\cos \theta_{j}, \sin \theta_{j}\right),
$$

with some constants $\left\{c_{m j}\right\}$.

Proof. The auxiliary proposition established in the proof of Theorem 1 actually proves the representation formula. Indeed, the proposition and (4) imply that the system $U_{i}\left(\theta_{k} ; \mathbf{x}\right), i=0, \ldots, n, k=i, \ldots, n$, is linearly independent and hence is a basis in $\Pi_{n}\left(\mathbb{R}^{2}\right)$.

To find the coefficients $c_{m j}$ in the above representation, we just compute

$$
\begin{aligned}
\mathcal{R}_{\theta}(P ; t) & =\sum_{j=0}^{n} \sum_{m=j}^{n} c_{m j} \mathcal{R}_{\theta}\left(U_{m}\left(\theta_{j} ; \cdot\right) ; t\right) \\
& =\sqrt{1-t^{2}} \sum_{m=0}^{n}\left\{\sum_{j=0}^{m} c_{m j} D_{m}\left(\theta_{j}-\theta\right)\right\} U_{m}(t) .
\end{aligned}
$$

Comparing this with the Chebyshev-Fourier expansion of $\mathcal{R}_{\theta}(P ; t)$, we arrive at the relation

$$
\sum_{j=0}^{m} c_{m j} D_{m}\left(\theta_{j}-\theta\right)=b_{m}(P ; \theta) .
$$

Therefore, given $P$ (and thus $\left\{b_{m}(P ; \theta)\right\}$ ), we determine $\left\{c_{m j}\right\}$ uniquely from the linear system

$$
\sum_{j=0}^{m} c_{m j} D_{m}\left(\theta_{j}-\theta_{k}\right)=b_{m}\left(P ; \theta_{k}\right), \quad k=0, \ldots, m .
$$

The proof is complete.

Clearly (13) is a representation of the form (12). We can get an $n$-term ridge representation of $P$ from (13) if we make $c_{n n}=0$. To do this, con- 
sider (14) for $m=n$. Using the translated directions $\left\{\theta_{j}+\varphi\right\}_{j=0}^{n}$ with a parameter $\varphi$ we would arrive at another representation of $b_{n}(P ; \theta)$ of the form (14) (with $m=n$ ), with some other coefficients $\left\{c_{m j}(\varphi)\right\}$, depending on $\varphi$, i.e., at

$$
\sum_{j=0}^{n} c_{n j}(\varphi) D_{n}\left(\theta_{j}+\varphi-\theta\right)=b_{n}(P ; \theta) .
$$

Next, setting $\theta=\theta_{k}+\varphi, k=0, \ldots, n$, we obtain the linear system

$$
\sum_{j=0}^{n} c_{n j}(\varphi) D_{n}\left(\theta_{j}-\theta_{k}\right)=b_{n}\left(P ; \theta_{k}+\varphi\right), \quad k=0, \ldots, n .
$$

By Cramer's rule,

$$
c_{n n}(\varphi)=\sum_{k=0}^{n} \beta_{k} b_{n}\left(P ; \theta_{k}+\varphi\right)
$$

with some constant coefficients $\left\{\beta_{j}\right\}$.

If $c_{n n}(0)=0$, then there is nothing to prove. So, assume in what follows that $c_{n n}(0) \neq 0$.

Consider first the case of $n$ odd. Since then $b_{n}(P ; \theta+\pi)=(-1)^{n} b_{n}(P ; \theta)$, we see that

$$
c_{n n}(\pi)=-c_{n n}(0) .
$$

Therefore, there exists an angle $\varphi \in(0, \pi)$ for which $c_{n n}(\varphi)=0$. This is a refinement of Schinzel's result: In [17], the existence of $n$ suitable directions was established while here we can obtain an $n$-term representation from any given system of directions $\left\{\theta_{j}\right\}$, just rotating it through a certain angle. Moreover, the form of the representation is given explicitly by (13).

We come to the same conclusion for $n$ even, provided the trigonometric polynomial $c_{n n}(\varphi)$ has a zero for some $\varphi$. But this is not always the case. For example, if $P$ is a radial function, say

$$
P(\mathbf{x})=F_{m}(\mathbf{x}):=\left(x^{2}+y^{2}\right)^{m}, \quad n=2 m,
$$

then $b_{n}\left(F_{m} ; \theta\right) \equiv$ const $\neq 0$.

As the next example shows, in case of $n$ even the above improvement of Schinzel's result need not hold.

Counterexample. Let $m \geq 1, n=2 m$, and $\theta_{1}<\ldots<\theta_{n}$ be any given set of directions such that $\theta_{n}-\theta_{1}<\pi / 2$. Then the polynomial $F_{m}=$ $\left(x^{2}+y^{2}\right)^{m}$ has no n-term representation by ridge polynomials with directions given by a rotation of $\theta_{1}, \ldots, \theta_{n}$. that

Assume the contrary. Then there exist ridge polynomials $\left\{r_{j}\right\}_{j=1}^{n}$ such

$$
\left(x^{2}+y^{2}\right)^{m}=\sum_{j=1}^{2 m} r_{j}\left(\mathbf{x} \cdot \xi_{j}\right),
$$


with $\xi_{j}:=\left(\cos \left(\theta_{j}+\varphi\right), \sin \left(\theta_{j}+\varphi\right)\right)$ and some $\varphi$. Since a rotation of the coordinate system does not change radial polynomials, we may suppose that $\varphi=0$ and $0<\theta_{1}<\ldots<\theta_{n}<\pi / 2$. Further, because $F_{m}$ is a homogeneous polynomial of degree $2 m$, the relation (15) implies that $r_{j}(t)=d_{j} t^{2 m}$ for all $j$. Thus, we have

$$
\sum_{j=1}^{2 m} d_{j}\left(x \cos \theta_{j}+y \sin \theta_{j}\right)^{2 m}=\left(x^{2}+y^{2}\right)^{m} .
$$

Comparing the coefficients of $x^{k} y^{2 m-k}$ on both sides, we get

$$
\left(\begin{array}{c}
2 m \\
k
\end{array}\right) \sum_{j=1}^{2 m} d_{j}\left(\cos \theta_{j}\right)^{k}\left(\sin \theta_{j}\right)^{2 m-k}= \begin{cases}0 & \text { for } k \text { odd } \\
\left(\begin{array}{c}
m \\
k / 2
\end{array}\right) & \text { for } k \text { even }\end{cases}
$$

Set

$$
\begin{aligned}
A_{j} & :=d_{j}\left(\sin \theta_{j}\right)^{2 m}, \quad t_{j}:=\cot \theta_{j}, \\
C_{2 k} & :=\left(\begin{array}{c}
m \\
k
\end{array}\right) /\left(\begin{array}{c}
2 m \\
2 k
\end{array}\right) \quad \text { for } k \text { even. }
\end{aligned}
$$

Clearly $\left\{0, A_{1}, \ldots, A_{2 m}\right\}$ satisfy the linear system

$$
\begin{aligned}
0 \cdot 1+A_{1}+\ldots+\quad A_{2 m} & =C_{0}, \\
0 \cdot t_{0}+A_{1} t_{1}+\ldots+A_{2 m} t_{2 m} & =0 \\
& \vdots \\
0 \cdot t_{0}^{2 m}+A_{1} t_{1}^{2 m}+\ldots+A_{2 m} t_{2 m}^{2 m} & =C_{2 m},
\end{aligned}
$$

with any $t_{0} \neq t_{1}, \ldots, t_{2 m}$. We choose $t_{0} \in\left(0, t_{1}\right)$. By Cramer's rule,

$$
0=\operatorname{det}\left[\begin{array}{cccc}
C_{0} & 1 & \ldots & 1 \\
0 & t_{1} & \ldots & t_{2 m} \\
C_{2} & t_{1}^{2} & \ldots & t_{2 m}^{2} \\
0 & t_{1}^{3} & \ldots & t_{2 m}^{3} \\
\ldots \ldots & \ldots & \ldots & \ldots . . \\
C_{2 m} & t_{1}^{2 m} & \ldots & t_{2 m}^{2 m}
\end{array}\right]=\sum_{k=0}^{m} C_{2 k} \operatorname{det} D_{k},
$$

where $D_{k}$ is obtained from the above matrix by deleting the first column and row $2 k$. It is known that the determinants $\operatorname{det} D_{k}$ are non-zero and of the same sign (since $t_{2 m}>\ldots>t_{0}>0$ ). In addition, all $C_{2 k}$ are positive numbers. Thus, we arrive at a contradiction. The proof is complete.

Having in mind the above arguments, it is easy to conclude that for $n$ even, every polynomial $P \in \Pi_{n}\left(\mathbb{R}^{2}\right)$ can be represented in one of the following forms: 
(i) $n$-term representation for a rotation of any given system of directions $\theta_{1}<\ldots<\theta_{n}$

(ii) radial function $+n$-term representation for any given system of directions $\theta_{1}<\ldots<\theta_{n}$.

To verify (ii) one needs to consider $P-\lambda F_{m}$ with a constant $\lambda$ chosen so that $c_{n n}\left(P-\lambda F_{m} ; \varphi\right)=0$ for some $\varphi$.

Acknowledgements. We thank the referee for a number of useful remarks and constructive suggestions.

\section{References}

[1] B. Bojanov and G. Petrova, Numerical integration over a disc. A new Gaussian quadrature formula, Numer. Math. 80 (1998), 39-59.

[2] - - - Uniqueness of the Gaussian cubature for a ball, J. Approx. Theory 104 (2000), 21-44.

[3] A. S. Cavaretta, C. A. Micchelli and A. Sharma, Multivariate interpolation and the Radon transform, Part I, Math. Z. 174 (1980), 263-279; Part II, in: Quantitative Approximation, R. DeVore and K. Scherer (eds.), Academic Press, New York, 1980, 49-62.

[4] M. E. Davison and F. A. Grunbaum, Tomographic reconstruction with arbitrary directions, Comm. Pure Appl. Math. 34 (1981), 77-120.

[5] C. F. Dunkl and Y. Xu, Orthogonal Polynomials of Several Variables, Cambridge Univ. Press, Cambridge, 2001.

[6] H. Hakopian, Multivariate divided differences and multivariate interpolation of Lagrange and Hermite type, J. Approx. Theory 34 (1982), 286-305.

[7] - Multivariate spline functions, B-splines basis and polynomial interpolation II, Studia Math. 79 (1984), 91-102.

[8] C. Hamaker and D. C. Solmon, The angles between the null spaces of X-rays, J. Math. Anal. Appl. 62 (1978), 1-23.

[9] F. John, Abhängigkeiten zwischen den Flächenintegralen einer stetigen Funktion, Math. Ann. 111 (1935), 541-559.

[10] B. Logan and L. Shepp, Optimal reconstruction of a function from its projections, Duke Math. J. 42 (1975), 645-659.

[11] R. Marr, On the reconstruction of a function on a circular domain from a sampling of its line integrals, J. Math. Anal. Appl. 45 (1974), 357-374.

[12] I. Mysovskikh, Interpolatory Cubature Formulas, Nauka, Moscow, 1981 (in Russian).

[13] K. Oskolkov, On representation of algebraic polynomials by superpositions of plane waves, Serdica Math. J. 28 (2002), 379-390.

[14] P. Petrushev, Approximation by ridge functions and neural networks, SIAM J. Math. Anal. 30 (1998), 155-189.

[15] J. Radon, Über die Bestimmung von Funktionen durch ihre Integralwerte längs gewisser Mannigfaltigkeiten, Ber. Verh. Sächs. Akad. 69 (1917), 262-277.

[16] T. J. Rivlin, Chebyshev Polynomials: from Approximation Theory to Algebra and Number Theory, 2nd ed., Wiley, New York, 1990.

[17] A. Schinzel, On a decomposition of algebraic polynomials in several variables, J. Théorie Nombres Bordeaux, 2003, to appear. 
[18] S. L. Sobolev, On formulas of mechanical cubature on the surface of a sphere, Sibirsk. Mat. Zh. 3 (1962), 769-791 (in Russian).

[19] D. C. Solmon, The X-ray transform, J. Math. Anal. Appl. 56 (1976), 61-83.

[20] B. A. Vostretsov and M. A. Kreĭnes, On the approximation of continuous functions by superpositions of plane waves, Dokl. Akad. Nauk SSSR 140 (1961), 1237-1240 (in Russian).

B. Bojanov

Department of Mathematics

University of Sofia

Blvd. James Boucher 5

1164 Sofia, Bulgaria

E-mail: boris@fmi.uni-sofia.bg
I. K. Georgieva

Institute of Mathematics Bulgarian Academy of Sciences

Akad. G. Bonchev St., bl. 8

1113 Sofia, Bulgaria

E-mail: irina@fmi.uni-sofia.bg

Received April 1, 2003

Revised version July 3, 2003 